\title{
A Study of ENSO Prediction Using a Hybrid Coupled Model and the Adjoint Method for Data Assimilation
}

\author{
Eli GALANTI AND ELI TZIPERMAN \\ Department of Environmental Sciences, Weizmann Institute of Science, Rehovot, Israel \\ MATthew Harrison AND ANTHONy Rosati \\ NOAA/GFDL, Princeton, New Jersey \\ ZIV SIRKES* \\ Institute of Marine Science, University of Southern Mississippi, Stennis Space Center, Mississippi
}

(Manuscript received 6 September 2002, in final form 7 April 2003)

\begin{abstract}
An experimental ENSO prediction system is presented, based on an ocean general circulation model (GCM) coupled to a statistical atmosphere and the adjoint method of $4 \mathrm{D}$ variational data assimilation. The adjoint method is used to initialize the coupled model, and predictions are performed for the period 1980-99. The coupled model is also initialized using two simpler assimilation techniques: forcing the ocean model with observed sea surface temperature and surface fluxes, and a 3D variational data assimilation (3DVAR) method, similar to that used by the National Centers for Environmental Prediction (NCEP) for operational ENSO prediction. The prediction skill of the coupled model initialized by the three assimilation methods is then analyzed and compared. The effect of the assimilation period used in the adjoint method is studied by using 3-, 6-, and 9-month assimilation periods. Finally, the possibility of assimilating only the anomalies with respect to observed climatology in order to circumvent systematic model biases is examined.

It is found that the adjoint method does seem to have the potential for improving over simpler assimilation schemes. The improved skill is mainly at prediction intervals of more than 6 months, where the coupled model dynamics start to influence the model solution. At shorter prediction time intervals, the initialization using the forced ocean model or the 3DVAR may result in a better prediction skill. The assimilation of anomalies did not have a substantial effect on the prediction skill of the coupled model. This seems to indicate that in this model the climatology bias, which is compensated for by the anomaly assimilation, is less significant for the predictive skill than the bias in the model variability, which cannot be eliminated using the anomaly assimilation. Changing the optimization period from 6 to 3 to 9 months showed that the period of 6 months seems to be a near-optimal choice for this model.
\end{abstract}

\section{Introduction}

Much progress has been made during the past decade both in developing a variety of models and theories for El Niño-Southern Oscillation (ENSO) (Neelin et al. 1998) and in developing data assimilation methods for the initialization of ENSO predictions (Latif et al. 1998). ENSO prediction models range from statistical models (e.g., Latif and Graham 1992), via intermediate reducedgravity models, such as that of Zebiak and Cane (1987),

* Deceased.

Corresponding author address: Eli Galanti, Environmental Sciences, Weizmann Institute of Science, Rehovot 76100, Israel. E-mail: eli.galanti@weizmann.ac.il to hybrid coupled models (e.g., Syu and Neelin 2000b), to fully coupled general circulation models (e.g., Rosati et al. 1997).

Prediction models need to be initialized using the observed data, and the choice of the method by which the observations are to be assimilated into the model is of great importance. Data assimilation methods used in tropical ocean models range from simple nudging techniques to sophisticated optimization methods like the Kalman filter and the 4D variational schemes (Ghil and Malanotte-Rizzoli 1991). Data insertion was used successfully in the Cane-Zebiak model, for example, where the observed winds were used to drive the ocean model (Cane et al. 1986). In the nudging method, the model fields are nudged toward the observed value, with a timescale depending on the assumed model and data 
errors (Chen et al. 1995; Kirtman and Zebiak 1997). Optimal interpolation (3D variational assimilation; Derber and Rosati 1989) is currently used operationally in the National Centers for Environmental Prediction (NCEP; Behringer et al. 1998). Finally, there are the Kalman filter and smoother (Ballabrera-poj et al. 2001; Cane et al. 1996; Miller and Cane 1989) and the 4D variational assimilation technique (adjoint method; e.g., Lee et al. 2000), which avoids some of the computational difficulties of the Kalman filter by not calculating the error covariance matrices.

Adjoint models are now used for 4D variational data assimilation in intermediate and complex models (e.g., Bennett and McIntosh 1982; Bennett et al. 1998; Thacker and Long 1988; Tziperman and Thacker 1989) and for operational weather prediction (Klinker et al. 2000). More specifically, the adjoint method of data assimilation has been used to initialize intermediate ENSO prediction models in the framework of ocean-only initialization (Bonekamp et al. 2001; Kleeman et al. 1995; Weaver et al. 2002) as well as in the framework of a coupled model initialization (Lee et al. 2000). These studies, while taking an important step toward the implementation of the adjoint method to ENSO prediction, were limited either by the simplicity of the dynamical models used or by the use of an ocean-only initialization.

Our objective in this paper is to study the potential of the adjoint method of data assimilation for ENSO prediction using a prediction system based on a hybrid coupled model and its adjoint. It is our hope that the use of a sophisticated assimilation method applied to a complicated and reasonably realistic ENSO model may lead to improvements in ENSO prediction skill. In order to estimate the performance of the adjoint assimilation in initializing the coupled model for ENSO prediction, we compare it to a three-dimensional variational data assimilation (3DVAR) method and to a simple nudging technique, all applied to the same model. The adjoint assimilation method is powerful yet quite complex. Since it has been hardly applied to the ENSO prediction problem, there is quite a bit of exploration to do in order to tune and optimize the adjoint method for this particular application. We start this tuning and optimization process in the present work, as follows: First, we examine the possibility of assimilating only the anomalous observed fields (the observed fields minus their seasonal climatology) in order to eliminate systematic climatological model bias, and the prediction skill is compared to that of the full-field assimilation. Next, we attempt to find the optimal time window over which the observed data is being assimilated by repeating the prediction experiments with assimilation periods of 3,6 , and 9 months.

The paper is organized as follows: in section 2 the hybrid coupled model and data used for the assimilation are described. The prediction experiments based on initialization with the forced ocean model (i.e., using in- sertion technique to assimilate the wind stress and nudging to assimilate SST) are presented in section 3, and the prediction experiments based on initialization with the 3DVAR method are presented in section 4 . The setup of the adjoint data assimilation is presented in section 5 , and the prediction experiments based on initialization with the adjoint method are then presented in section 6. We conclude in section 7.

\section{The model and data}

The coupled ocean-atmosphere model we use, and its adjoint, were described in detail by Galanti et al. (2002), who used the model for an adjoint sensitivity study of the coupled ocean-atmosphere instability dynamics (see also Galanti and Tzipermann 2003). We therefore give here only a brief description of the model.

The ocean model is based on the Geophysical Fluid Dynamics Laboratory (GFDL) Modular Ocean Model (MOM; Pacanowski and Griffies 1999). The model domain is the Indo-Pacific region, $50^{\circ} \mathrm{S}-50^{\circ} \mathrm{N}, 30^{\circ} \mathrm{E}-70^{\circ} \mathrm{W}$. The model resolution is $3^{\circ}$ in longitude, $3^{\circ}$ going to $1^{\circ}$ at the equator in latitude, and 30 depth levels, where the top 15 layers are within the top $200 \mathrm{~m}$ of the ocean. The model uses a modified Richardson number-dependent vertical mixing scheme (Pacanowski and Philander 1981; Syu and Neelin 2000a) and constant horizontal viscosity and diffusivity.

The statistical atmospheric model is based on a singular vector decomposition (SVD) using the NCEP SST (Reynolds and Smith 1994) and the wind stress and heat fluxes of the European Centre for Medium-Range Weather Forecasts (ECMWF) reanalysis (Gibson et al. 1997). Only the first three SVD modes are used (Harrison et al. 2002). The coupling of the atmospheric variables to the anomalous SST is done between $20^{\circ} \mathrm{S}$ and $20^{\circ} \mathrm{N}$, while outside of this area only the climatological atmospheric fields are used to force the ocean.

The coupled model is run as follows: at each time step, the SST anomalies are calculated with respect to the monthly climatological SST of the uncoupled ocean model. Next, the wind stress and heat flux anomalies are derived from the SST anomalies using the statistical atmospheric model. Finally, the calculated wind stress and heat flux anomalies are added to the monthly climatological Florida State University (FSU) wind stress and to the climatological model heat flux, respectively, to be used as forcing for the ocean model.

The model exhibits interannual variability similar to although weaker than, the observed ENSO variability (Galanti et al. 2002). The simulated SST anomalies are mainly in the east and central Pacific, the oscillation timescale is $3-4 \mathrm{yr}$, and the maximum of the warm events is reached during November-December, in a reasonable agreement with observations.

As the main goal of this study is to try to understand the effect of the adjoint assimilation on the prediction skill of the coupled model, for the data to be assimilated 
(a) observations

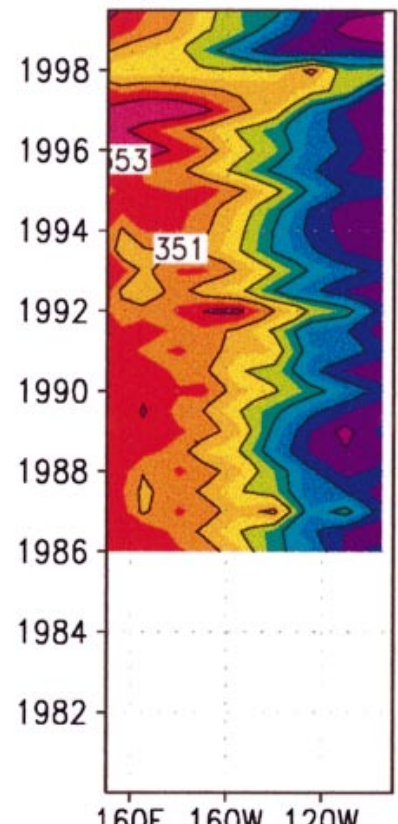

160E 160W 120W (b) simulation

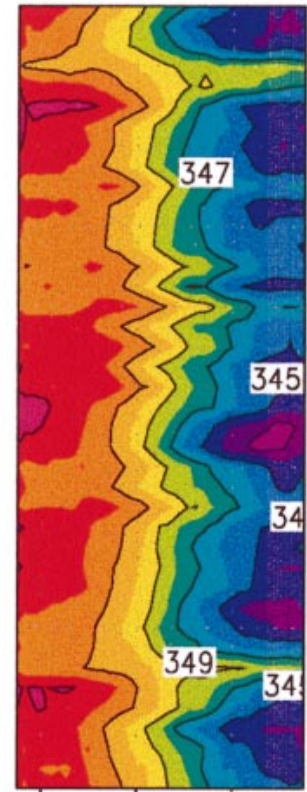

160E 160W 120W (c) 3Dvor

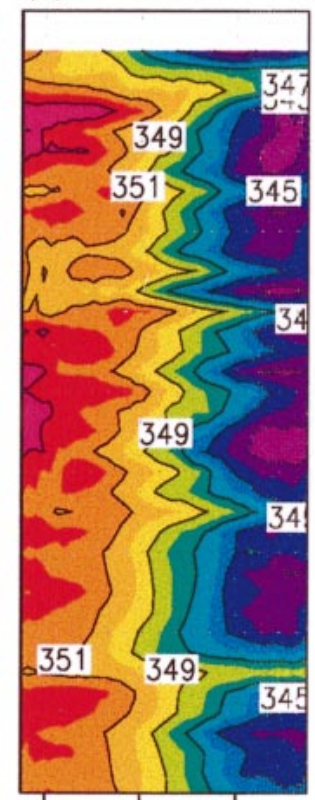

160E 160W 120W (d) odjoint

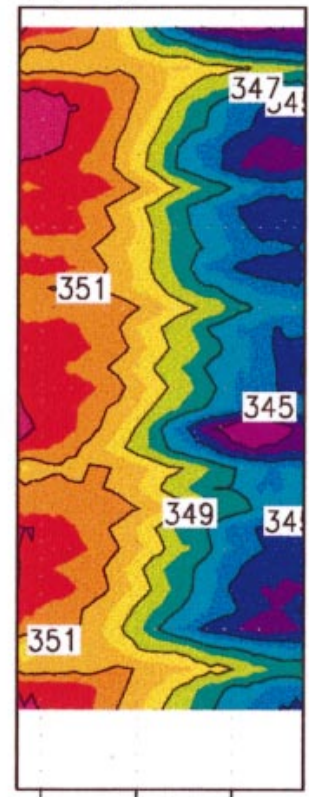

160E 160W 120W

FIG. 1. The ocean model heat content (in $10^{9} \mathrm{~J} \mathrm{~m}^{-2}$ ) along the equator, integrated from the surface to a depth of $250 \mathrm{~m}$, as obtained from the different initialization schemes: (a) the observed heat content (TAO array), (b) initialization using the forced ocean model, (c) initialization using the 3DVAR, and (d) initialization using the adjoint assimilation. Contour interval is $2 \times 10^{9} \mathrm{~J} \mathrm{~m}^{-2}$. The adjoint initialization produces initial conditions every 6 months (in Jan and $\mathrm{Jul}$ ), and all four plots are therefore given at this temporal resolution.

we chose the three most necessary variables for describing ENSO's dynamics and evolution: the SST, the subsurface temperatures (which reflect the thermocline depth), and the winds. The SST data used here are the weekly NCEP SST (Reynolds and Smith 1994), the subsurface temperatures are the daily Tropical Atmosphere Ocean (TAO) mooring observations (McPhaden et al. 1998), and the winds are the monthly NCEP reanalysis (Kalnay et al. 1996). The data cover the period between 1980 and 1999.

\section{Prediction with initial conditions from a forced ocean-only simulation}

The focus of this study is the comparison of the predictive skill based on initialization using the adjoint method with less complicated methods of data assimilation and initialization. The first initialization considered here is using a forced ocean run as follows: The ocean model, after being spun up, was forced with the NCEP winds, and its SST was restored to the NCEP SST with a 5-day restoring timescale. The model state was saved at the beginning of each month to serve as the initial conditions for ENSO prediction; this type of initialization will be referred to as the "simulation run." Figure 1 shows the observed heat content for the initial conditions (integrated from the surface to a depth of 250 $\mathrm{m})$ along the equator, together with the ocean model heat content obtained using the simulation (Fig. 1b). Also shown are the 3DVAR (Fig. 1c) and the adjoint assimilation (Fig. 1d), both described below. Only the heat content initial conditions for January and July are shown, because initial conditions for other months were not calculated with the adjoint method (see section $5 b$ ). It can be seen that the initial conditions produced by the simulation run (Fig. 1b) capture some of the observed features of the subsurface temperature along the equator, but in some cases (e.g., 1990-95) it fails to produce the observed subsurface temperature.

Figure 2a shows 2-yr segments of the Niño-3 index as predicted by the coupled model initialized by January initial conditions from the forced ocean simulation run compared to the observed Niño-3 index. The correlation of the predicted Niño-3 index with the observed is shown in Fig. 3a (dashed line) together with the observed persistence (thick solid line). For the 18 members used for the skill analysis, the 95\% confidence level is 0.4 , putting a threshold on the significance of the presented correlation. The rms error of the prediction (dash) together with the rms error of the persistence (thick solid) is shown in Fig. 3b (dashed line). The prediction skill for the first 5 months is similar to that of the persistence, suggesting that the coupled model dynamics are not very influential on this timescale. At time intervals longer than 6 months, the skill of persistence drops, while the model prediction shows a correlation 

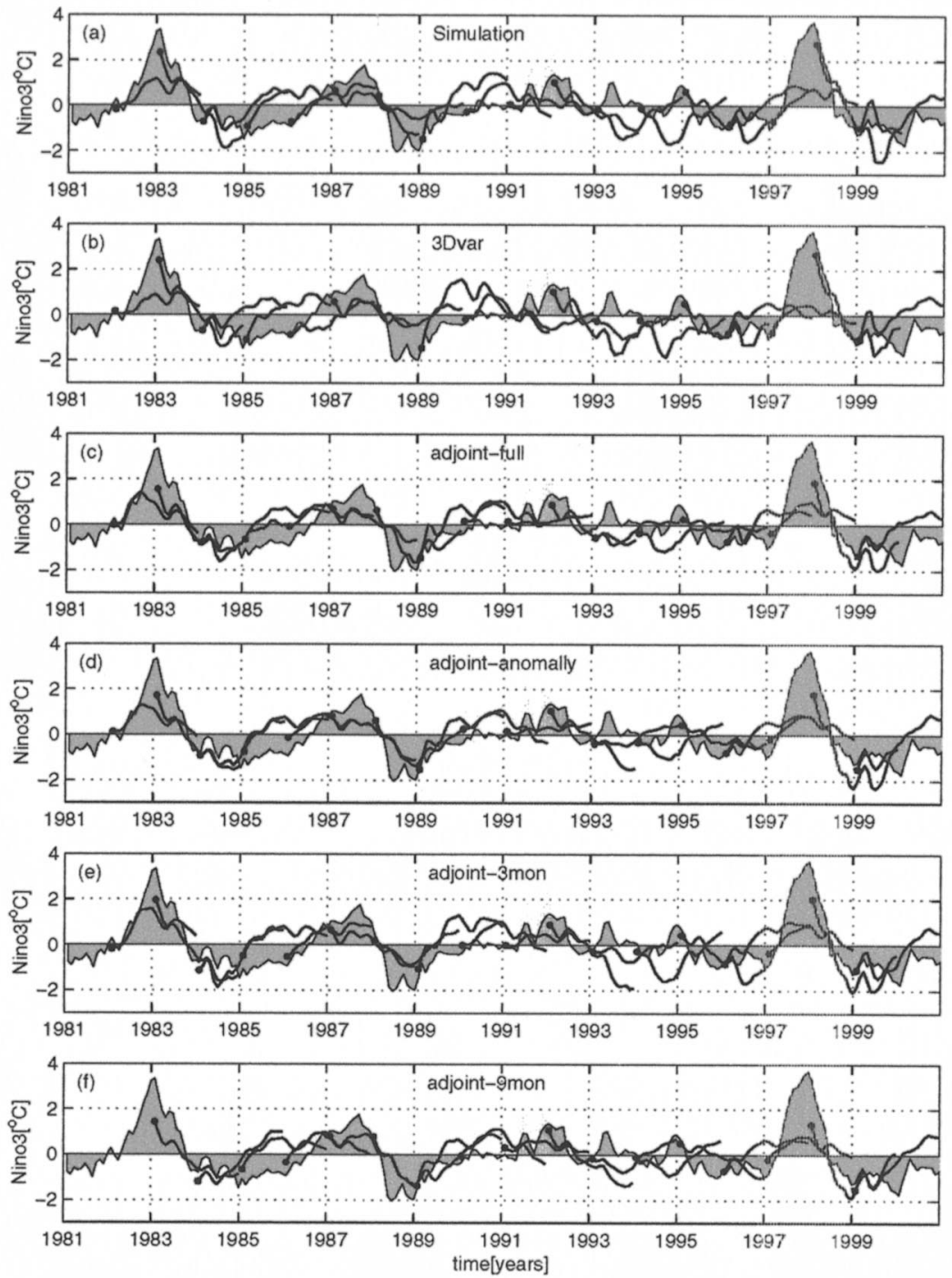

FIG. 2. The Niño-3 index as predicted by the coupled model (thick segments) on top of the observed Niño-3 index (shaded area); each segment shows $2 \mathrm{yr}$ of prediction, and the beginning of each prediction is marked with a filled circle. Shown are the predictions done with initial conditions obtained from the different initialization schemes: (a) simulation (section 3), (b) 3DVAR (section 4), (c) adjoint-full fields (section 6a), (d) adjoint-anomaly assimilation (section 6c), (e) adjoint-3-month optimization period (section 6d), and (f) adjoint-9-month optimization period (section 6d).

of about 0.5 ; surprisingly, this skill does not drop and remains at that level for the duration of the 2-yr prediction. As will be seen later, this characteristic does not change when using different initialization schemes. The rms error of the predictions shows a behavior consistent with the correlation and reinforces the observation that the model prediction skill does not drop significantly at longer lead times. The prediction skill with initialization in July (Figs. 4a and 5a,b) shows a similar picture, except that the skill (of both model and persistence) drops after about 8 months at late winter of the second year (McPhaden et al. 1998).

\section{Prediction with initial conditions obtained from a 3DVAR}

The next assimilation method to be considered is the 3D variational assimilation, also known as optimal interpolation (Derber and Rosati 1989; Rosati et al. 1997), which is similar to the method used today for the NCEP ENSO operational prediction initialization (Behringer et al. 1998). All assimilation methods are applied to the same ocean model in order to enable a consistent comparison of the assimilation and prediction skills. The 3DVAR scheme uses the same SST and wind products 
(a) Correlation

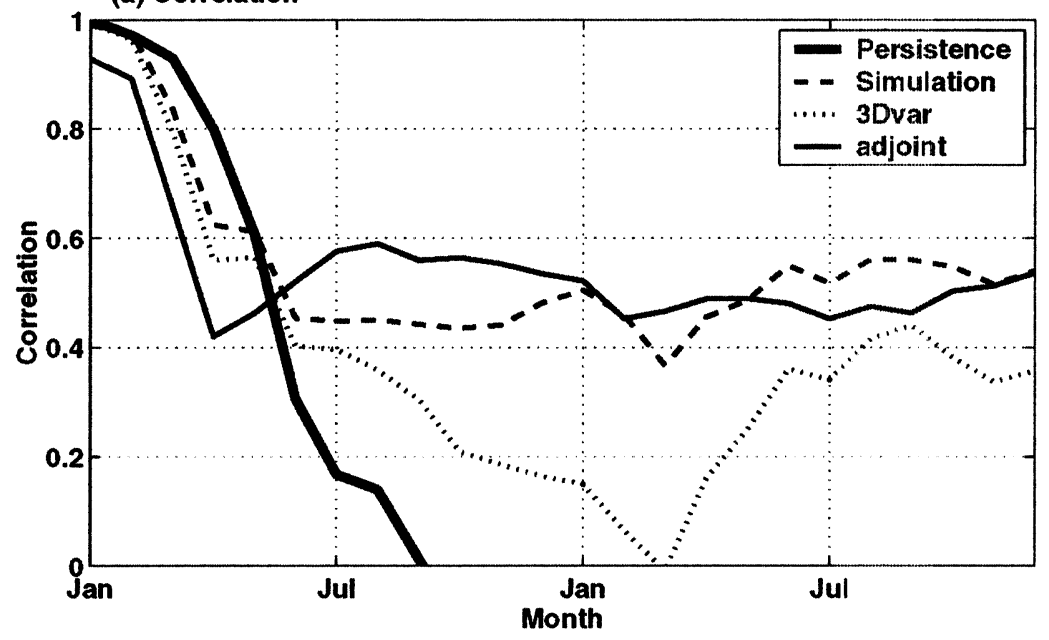

(b) RMS error

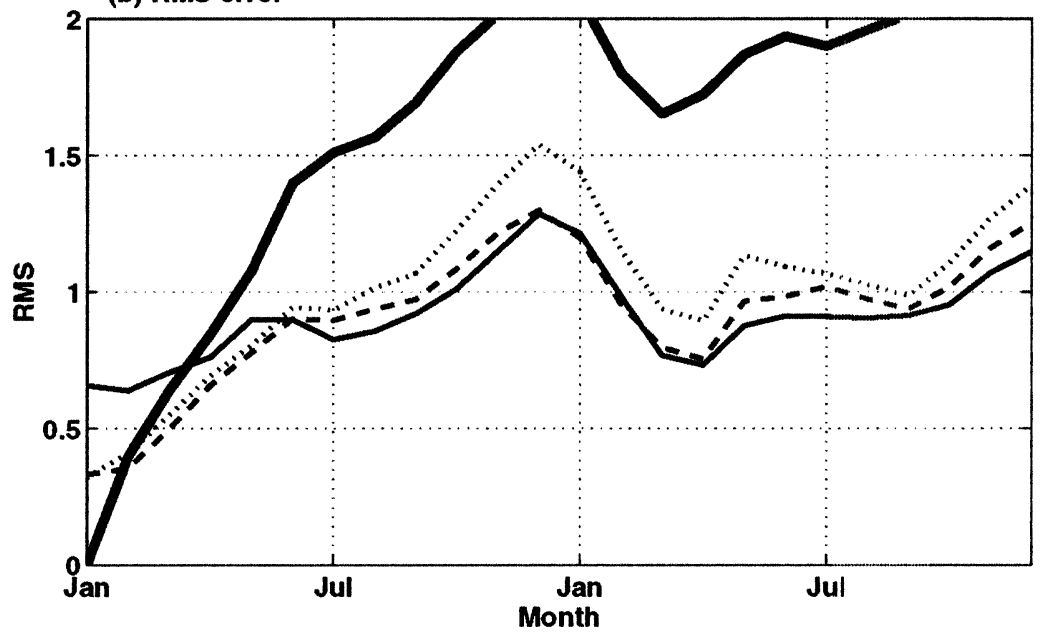

FIG. 3. Prediction skill of the coupled model for the different initialization schemes. (a) Correlation between the predicted and the observed Niño-3 as function of the month of prediction. (b) The rms error of the predicted Niño-3. Shown are the calculated persistence (thick solid line), prediction with simulation initial conditions (dashed line), prediction with 3DVAR initial conditions (dotted line), and prediction with adjoint initial conditions (thin solid line).

as the simulation, as well as the TAO subsurface temperature (see section 2). As with the simulation initialization, the 3DVAR scheme initializes the ocean model forced by the observed winds.

Figure $2 b$ shows the 2-yr segments of the Niño-3 index as predicted by the coupled model with the 3DVAR January initializations. The correlation of the predicted Niño-3 index with the observed one is showed in Fig. 3a (dotted line), and the rms error of the prediction is shown in Figs. $3 b$ (dotted line). During the first 5 months of the prediction, there is no difference between the skill of the simulation-based prediction (dash) and that of the 3DVAR-based prediction (dots); both are similar to persistence (thick solid). From 6 months till the end of the prediction, the 3DVAR has a lower correlation and a larger rms error compared to the simulation-based prediction. The same behavior is seen in the predictions starting in July (Figs. 4c and $5 \mathrm{a}, \mathrm{b}$, dotted lines), although to a lesser extent; the correlation drops more than the simulation-based prediction, and the rms error is larger.

Consider the effect of the 3DVAR on the equatorial heat content initial conditions (Fig. 1c) in comparison to the initial conditions obtained from the simulation run (Fig. 1b) and the observed temperature (Fig. 1a). The assimilation of the subsurface temperature into the model forces the model temperature to get closer to the observed subsurface temperature more than in the sim- 

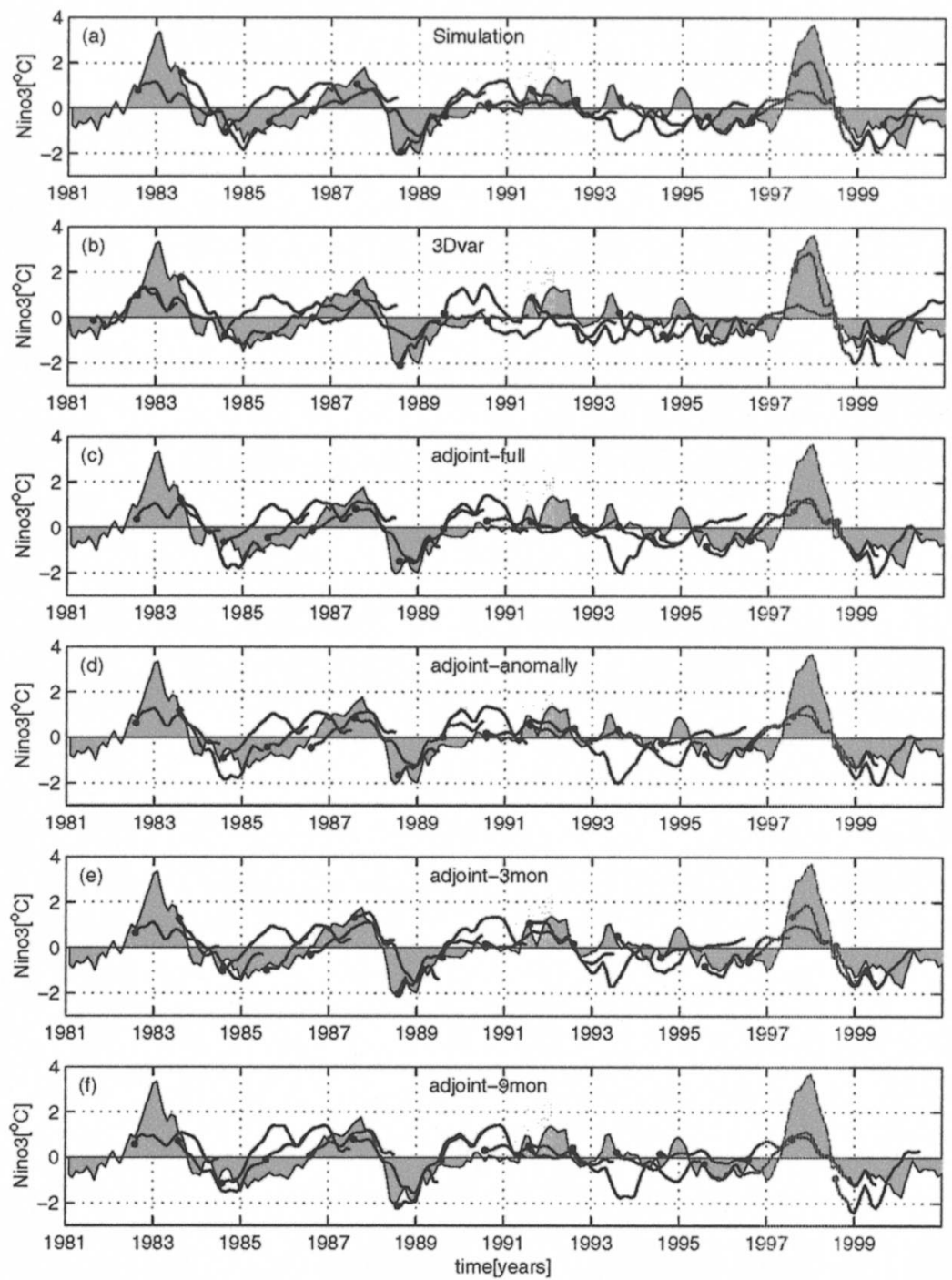

FIG. 4. Same as Fig. 2, but for predictions from Jul initial conditions.

ulation run, where the model is forced with surface fluxes only. The analysis obtained from applying the 3DVAR to our model (Fig. 1c) is similar to that obtained by Rosati et al. (1997, their Fig. 1). Nevertheless, even within the 6-month prediction period, where the initial ocean state dominates the skill, the 3DVAR does not improve the prediction skill of the coupled model over the simulation-based initial conditions. If therefore seems that the assimilation of the SST into the ocean model is sufficient for forcing both the surface and the subsurface temperature fields that influence the Niño-3 index during the first 6 months of prediction. Beyond the first 6 months of the prediction, the coupled model dynamics come into play, and there the assimilation of the subsurface temperature proves to actually degrade the prediction skill. A possible reason for this behavior is the inconsistency of the initial conditions calculated by the 3DVAR with the coupled model dynamics (see section $6 \mathrm{~b}$ ).

The above possible explanations for the low predictions skill obtained with 3DVAR initialization should be considered especially with respect to the results of Rosati et al. (1997), wherein the skill of the ENSO prediction based on the same 3DVAR initialization scheme was much better than in our case. There are several possible reasons for this discrepancy: One major difference is that the atmospheric model of Rosati et al. (1997) is a general circulation model, while ours is statistical. Another important difference is that their ocean model's horizontal resolution is 3 times finer than ours. These differences and their effect on the prediction skill justify further study, but that is out of the scope of the 
present work. Finally, we note that Rosati et al. (1997) study the predictability for the period 1979-88, while our prediction period is $1980-89$. We have calculated the skill of our model for the 1980-88 predictions (not shown), and the skill remained relatively low, so the slight difference in the period considered does not seem to be significant.

\section{Setup of the adjoint assimilation}

In this section we describe the setup of the coupled model initialization using the adjoint method (4D variational assimilation). [For further discussion of the adjoint method of data assimilation see, e.g., Tziperman and Thacker (1989), Tziperman et al. (1992a), or Marotzke (1992)].

\section{a. The cost function}

The adjoint method of data assimilation is based on the minimization of a cost function, which is a measure of the distance between the model solution and the observations, over a certain spatial and temporal domain. The cost function used in this study is based on the square of the difference between the observations and the model solution for the temperature $(T), \mathrm{SST}$, and wind stress components $(\tau)$. Each contribution to the cost function is weighted according to the relevant error estimates (Tziperman et al. 1992a):

$$
\begin{aligned}
\mathbf{J}= & \sum_{i} W_{i}^{\mathrm{TAO}}\left[T_{i}^{\mathrm{TAO}}-A_{i}\left(T^{\mathrm{model}}\right)\right]^{2} \\
& +\sum_{j} W_{j}^{\mathrm{REYNOLDS}}\left[\mathrm{SST}_{j}^{\mathrm{REYNOLDS}}-B_{j}\left(\mathrm{SST}^{\mathrm{model}}\right)\right]^{2} \\
& +\sum_{k} W_{k}^{\mathrm{NCEP}}\left[\tau_{k}^{\mathrm{NCEP}}-C_{k}\left(\tau^{\text {model }}\right)\right]^{2},
\end{aligned}
$$

where each of the indices $i, j$, and $k$ represent the summation over the spatiotemporal domain of the observations (and wind stress vector components), and the matrices $A_{i}, B_{j}$, and $C_{k}$ transform the model variables into the observations' spatiotemporal coordinates $i, j$, and $k$ (Derber and Rosati 1989). The model temperature and wind are bilinearly interpolated to the observational locations from the nearest horizontal points. In the vertical, the observations are interpolated into the model grid.

The weights for the TAO mooring terms are (following Tziperman et al. 1992b)

$$
W_{i}^{\mathrm{TAO}}=\frac{d x_{i} d y_{i} d z_{i}}{L_{\mathrm{hor}}^{\mathrm{TAO}} L_{\mathrm{ver}}^{\mathrm{TAO}}} \frac{1}{W_{s}^{\mathrm{TAO}}} \frac{1}{\left(E_{i}^{\mathrm{TAO}}\right)^{2}},
$$

where $d x_{i} d y_{i} d z_{i}$ is the model grid volume element, $L_{\mathrm{hor}}^{\mathrm{TAO}}=400 \mathrm{~km}$ and $L_{\mathrm{ver}}^{\mathrm{TAO}}=2 \cdot d z_{i}$ are estimated horizontal and vertical correlation length scales, $W_{s}^{\text {TAO }}=1$ (nondimensional) is the reciprocal of the observation sampling rate (which is $1 /$ day) normalized by 1 day, and $E_{i}^{\mathrm{TAO}}$ is the estimated error of the observations. The observational error estimate is set to be the standard deviation,

$$
E_{i}^{\mathrm{TAO}}=\operatorname{STD}\left(T_{i}^{\mathrm{TAO}}-\tilde{T}_{i}^{\mathrm{TAO}}\right),
$$

where $\tilde{T}_{i}^{\mathrm{TAO}}$ is the observed temperature smoothed by a running average of 3 months. This error estimate is simply the standard deviation of the high-frequency part of the observed temperature. The high-frequency variability of the observations cannot be resolved by the hybrid coupled model and is therefore treated as a combined observational/model error.

The weights for the Reynolds SST terms are set as

$$
W_{j}^{\mathrm{REYNOLDS}}=\frac{d x_{j} d y_{j}}{L_{\mathrm{hor}}^{\mathrm{REYNOLDS}}} \frac{1}{W_{s}^{\mathrm{REYNOLDS}}} \frac{1}{\left(E_{j}^{\mathrm{REYNOLDS}}\right)^{2}},
$$

where $L_{\text {hor }}^{\text {REYLDS }}=400 \mathrm{~km}$ is a horizontal correlation scale, $W_{s}^{\mathrm{REYNOLDS}}=1 / 7$ (nondimensional) is the reciprocal of the weekly observational sampling rate normalized by 1 day, and $E_{j}^{\text {REYNOLDS }}$ is the estimated error of the observations. The SST error estimate is set to be

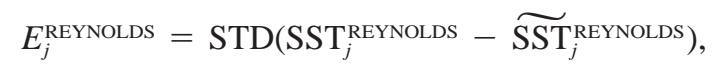

where $\widetilde{\mathrm{SSJ}}_{j}^{\mathrm{REYNOLDS}}$ is again the observed SST smoothed by a running average of 3 months. The weights for the NCEP wind stress terms are

$$
W_{j}^{\mathrm{NCEP}}=\frac{d x_{j} d y_{j}}{L_{\mathrm{hor}}^{\mathrm{NCEP}}} \frac{1}{W_{s}^{\mathrm{NCEP}}} \frac{1}{\left(E_{j}^{\mathrm{NCEP}}\right)^{2}},
$$

where $L_{\mathrm{hor}}^{\mathrm{NCEP}}=1000 \mathrm{~km}$ is an estimated horizontal correlation scale, $W_{s}^{\text {NCEP }}=1 / 30$ (nondimensional) is the reciprocal of the observations' sampling rate normalized by 1 day, and $E_{j}^{\text {NCEP }}=0.1 \mathrm{dyn}^{-2}$ is the estimated error of the observations.

\section{b. Optimization and prediction procedures}

In this study, we choose to regard the model dynamics as strong constraints to the assimilation; that is, we do not try to correct the model dynamics but optimize only the model initial conditions. There are advantages and disadvantages to the strong constraints assumption, as well as to the weak constraints assumption, wherein one optimizes some aspects of the model dynamics as well (Bennett 1992). If one's goal is to obtain an optimal estimate of the ocean state, then the weak constraints assumption is perhaps more suitable since it allows for the ocean dynamics to be adjusted to compensate for model errors. On the contrary, if the goal is to predict the state of the equatorial Pacific ocean-atmosphere system, then the strong constraints assumption may be preferable: with the weak constraints assumption, the model dynamics (mixing parameters, surface fluxes, etc.) are being adjusted during the assimilation, yet we have no knowledge about how to correct the model dynamics during the prediction phase. The result may be an "initial shock" that the coupled model will exhibit in the transition from assimilation to prediction periods. This 


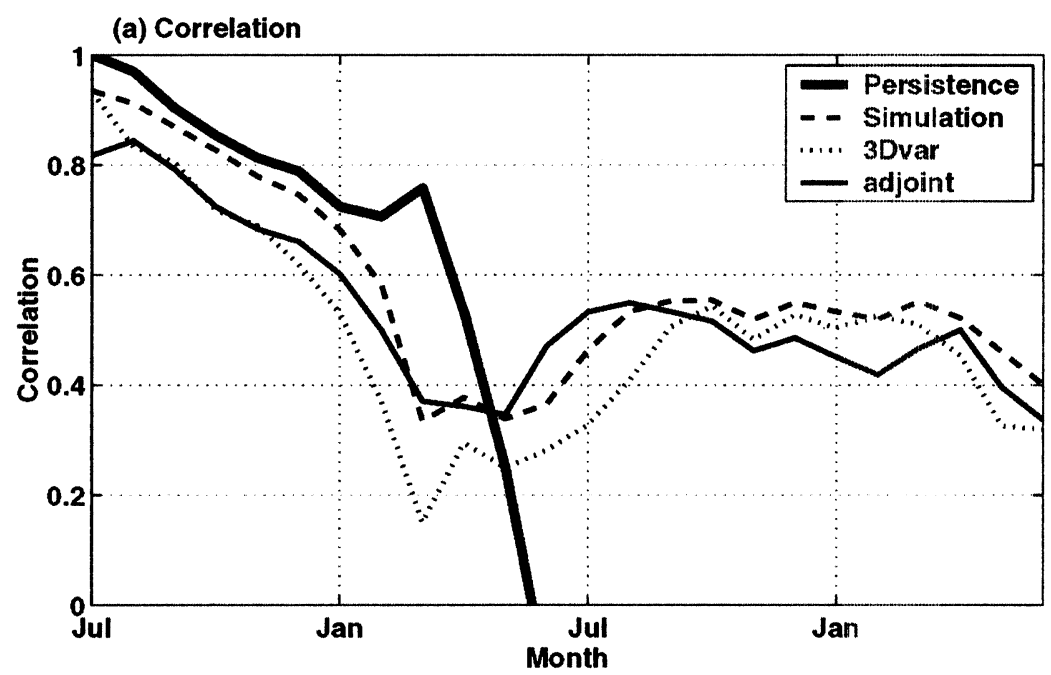

(b) RMS error

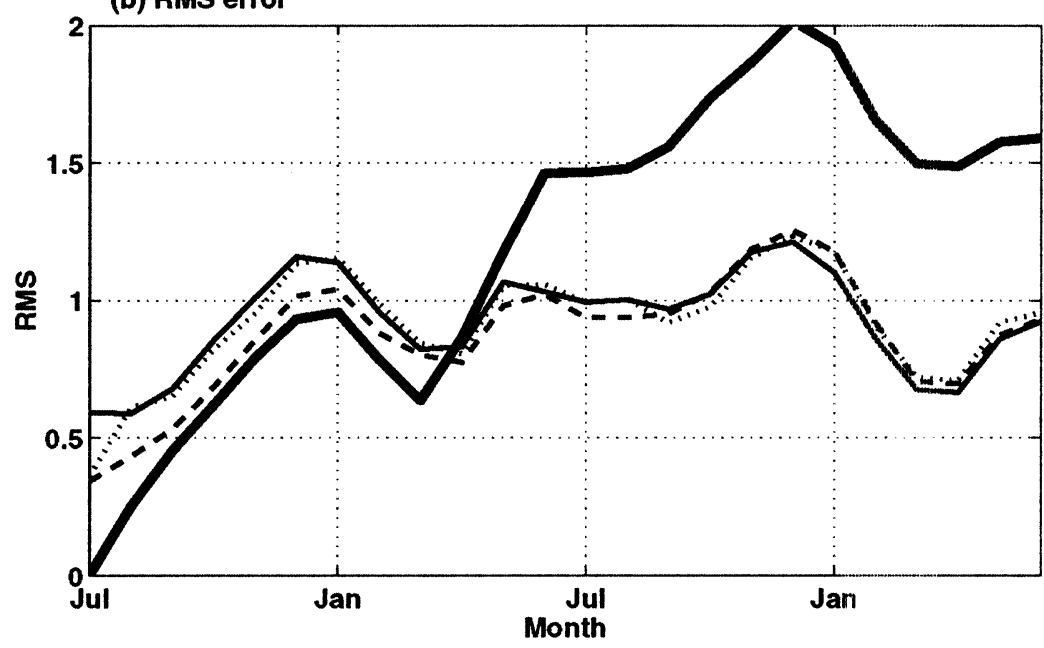

FIG. 5. Same as Fig. 3, but for predictions from Jul initial conditions.

can be avoided by using the strong constraints assumption, wherein the same model dynamics are used throughout.

Furthermore, we optimize the initial conditions for the temperature field only. The salinity field is a less important dynamical variable for the equatorial Pacific than the temperature, and the velocity field is expected to adjust to the temperature field within about 1 day and therefore need not be optimized (Rosati et al. 1997; Sirkes et al. 1996). [A recent study by Burgers et al. (2002) suggested that close to the equator, currents may, in fact, need to be assimilated and optimized.] The initial guess for the temperature initial conditions to be calculated by the adjoint iterative minimization is taken as the analysis obtained from the simulation run.

The optimization itself is done using a quasi-Newton limited memory BFGS algorithm for the minimization of the cost function (Gill et al. 1981), and we run the optimization for 10 iterations, which was found to be sufficient for satisfactory convergence (section 6). The adjusted optimal initial conditions for the temperature are then used to run the coupled model again for the assimilation time interval to obtain the initial conditions for the prediction.

Two approaches to the adjoint methods of data assimilation are examined here. In the first approach, the full observed and model fields are being used in the cost function, while, in the second, only anomaly fields with respect to the monthly climatology are used to calculate the cost function. In the first approach, the cost function is constructed from the difference between the observed fields and the model fields so if a seasonal or time-independent climatological bias exists in the model, the assimilation process will try to correct this bias using the observed fields. Correcting the model climatological bias in a way that contradicts the model 
dynamics, however, may have some undesired effects on the prediction skill, as explained below. In the second approach, the climatology of each field is therefore subtracted (i.e., the model climatology is subtracted from the model solution, and the observed climatology is subtracted from the observed fields). The cost function is now composed of the difference between the anomalous fields so the climatological model bias does not affect the assimilation anymore.

Another issue that will be discussed below (section $6 \mathrm{~d})$ is the time period over which the assimilation is performed. Throughout the paper we will use a time interval of 6 months unless indicated otherwise.

\section{ENSO prediction based on adjoint assimilation}

In this section we initialize the coupled model prediction runs with initial conditions obtained from the adjoint assimilation scheme. Initial conditions are obtained from an adjoint optimization performed over the 6 months prior to the time of the initialization, and the cost function is based on the full observed and model fields. The initial conditions based on the adjoint assimilation (Fig. 1d) are less close to the observations (Fig. 1a) than those obtained from the 3DVAR (Fig. 1c), which is to be expected, as discussed above.

The behavior of the cost function and its components during the optimization is shown in Fig. 6a. The cost function shown is an average over all the January and July 6-month adjoint assimilation periods. The cost function value is reduced by a factor of 2 after 10 iterations, and the rate of convergence decreases substantially toward the 10th iteration. This may not seem to be a large reduction, but note that we start the optimization with a fairly good initial guess for the optimal initial conditions, obtained from the forced simulation runs, so we do not expect the optimization to significantly reduce the cost from its initial value. The averaged contribution to the final cost function by the SST penalty terms in (1) is about $60 \%$, the contribution by the TAO array data is about $30 \%$, and the wind penalty terms contribute about $10 \%$. The relative contributions of the SST and the TAO array to the cost function are different during the 1980 s, when most of the cost function is determined by the SST data-model misfit, while during the 1990s, when the TAO array expanded, the contributions to the cost function by the SST and the TAO array are about the same. Another interesting aspect of the optimization is that the model winds are hardly being optimized (their penalty term is not reduced much by the optimization). There are two reasons for that: one is that the relative contribution of the winds to the cost function is small. The second reason is that the winds derived from the statistical atmospheric model depend on the model SST in a simple and perhaps not very realistic manner. Optimizing the wind therefore implies that the model SST would need to be corrected in a way that deviates from the observed SST. That, in turn, will result in a large increase of the cost function due to the direct SST penalty terms. The optimization therefore prefers the optimization of the SST over the optimization of the winds, explaining the lack of significant reduction in the winds' penalty terms. The absolute value of the cost function gradient with respect to the temperature initial conditions decreases during the optimization by a factor of 4 (Fig. 6b), when most of the reduction occurs during the first 4 iterations.

While we used the adjoint method to initialize the coupled model, we used the 3DVAR and simulation methods to initialize the ocean-only model. This choice was made with the notion that a coupled model initialization with the adjoint method will eventually lead to better prediction for the reasons discussed below. However, as we wanted to compare the performance of the adjoint method to that used for operational prediction at present, the 3DVAR procedure we use follows that of Rosati et al. (1997), who initialized the ocean-only model. This 3DVAR procedure specifies the wind and therefore cannot be used with a coupled model.

In the following subsections we discuss several variations in the procedure of the adjoint data assimilation approaches used to initialize the coupled model. We also study the 1997/98 ENSO event, as predicted by the coupled model initialized by the different adjoint assimilation variants.

\section{a. Assimilation using the full observed fields}

Consider first the prediction skill when the full observed fields are used in the cost function for January initial conditions (Figs. $2 \mathrm{c}$ and $3 \mathrm{a}, \mathrm{b}$ ). In the first 5 months, the correlation between the predicted Niño-3 and the observed Niño-3 is smaller than that of the prediction based on initial conditions calculated by the simulation run, simply because the adjoint initialization takes into account the model dynamics that prevent the model from getting as close to the observations, as in the case of the forced simulation run (section 3; Fig. $3 a)$. During months 6-12 of the prediction, when the model dynamics come into play, the adjoint-based prediction has a somewhat better correlation with observations than the simulation-based prediction; however, the rms error of the prediction is similar in both cases (Fig. 3b). The results from the predictions with July initial conditions (Figs. 4c and 5a,b) show a somewhat different picture. During the first 6 months the adjointbased prediction is again, as for the January initial conditions, less successful than the simulation-based prediction. However, at longer times, the skill of the adjoint-based prediction is similar to that of the simulation-based prediction and not better as it was for the January initial conditions. This behavior may be a result of the "spring barrier" (Webster 1995); that is, by the time the model dynamics start to play an important role, the spring barrier erases the additional skill due to the 
(a)

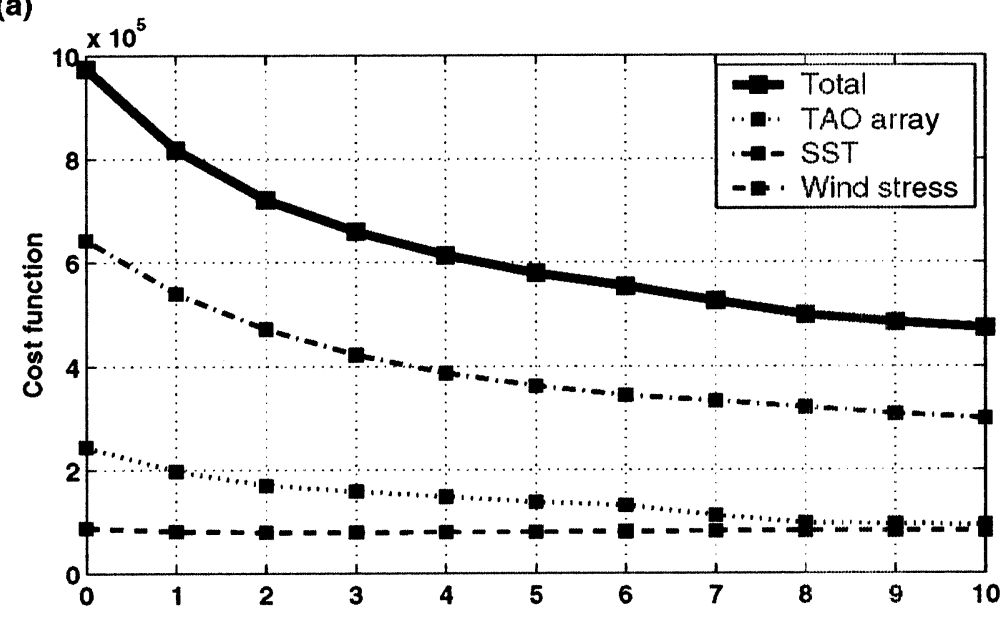

(b)

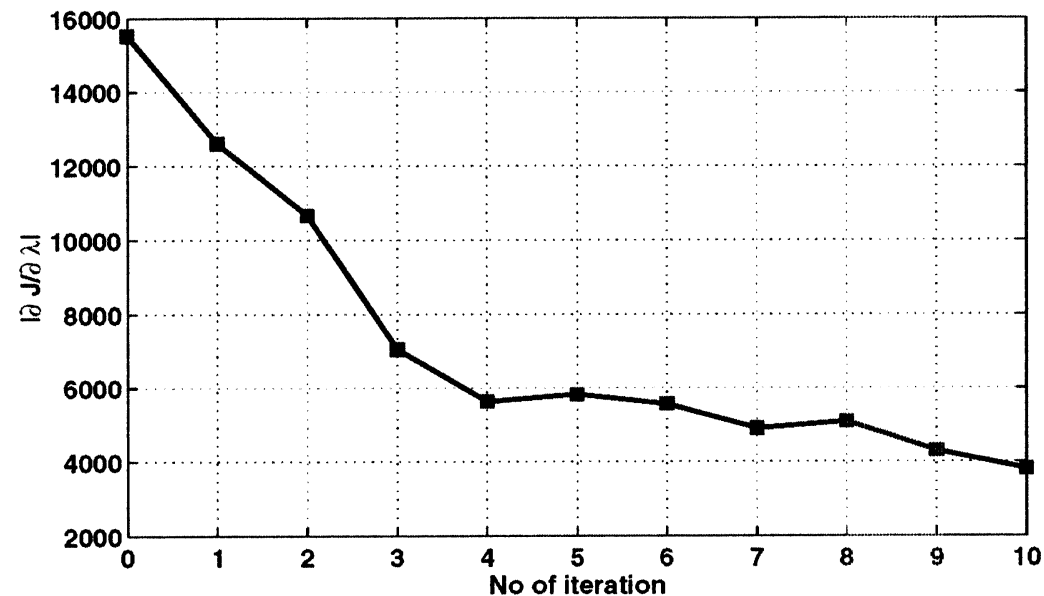

FIG. 6. (a) The reduction in the cost function value and its components as a function of the number of iterations (an average over all the Jan and Jul 6-month period adjoint initializations). The dotted line, dash-dotted line, and dashed line show the contribution of the TAO array, SST, and wind stress to the cost function, respectively. (b) The reduction in the norm of the cost function gradient as a function of the number of iterations.

hopefully superior initial conditions calculated by the adjoint method.

The main point of this section is that the adjoint initialization does seem to have the potential for improving the prediction skill of the coupled model, as shown in the case of the January initialization and by the comparison to the 3DVAR scheme. We note that the discrepancies between our results for the 3DVAR initialization and those of Rosati et al. (1997) raise important questions regarding the effects of the model used on the prediction skill. Therefore, a better evaluation of the prediction skill based on adjoint initialization, especially compared to the 3DVAR, requires using the higher resolution used in operational prediction, a task that is beyond the scope of the present study.

\section{b. The $1997 / 98$ event}

Let us now focus on the 1997/98 event and compare the predictions from initial conditions obtained from the three different assimilation schemes. The 1997/98 event was suggested to have been partially triggered by westerly wind bursts (WWB) after January 1997 (e.g., van Oldenborgh 2000). The predicted ENSO event in our model, lacking the WWB, is therefore expected to be of a smaller amplitude than the observed event.

Figure 7 shows the temperature at $0^{\circ}, 140^{\circ} \mathrm{W}$ as function of depth, as predicted by the coupled model initialized with the three assimilation methods: the simulation, 3DVAR, and adjoint. Also shown is the observed temperature at that location. Looking at the predicted temperature during December 1997, we see that the 

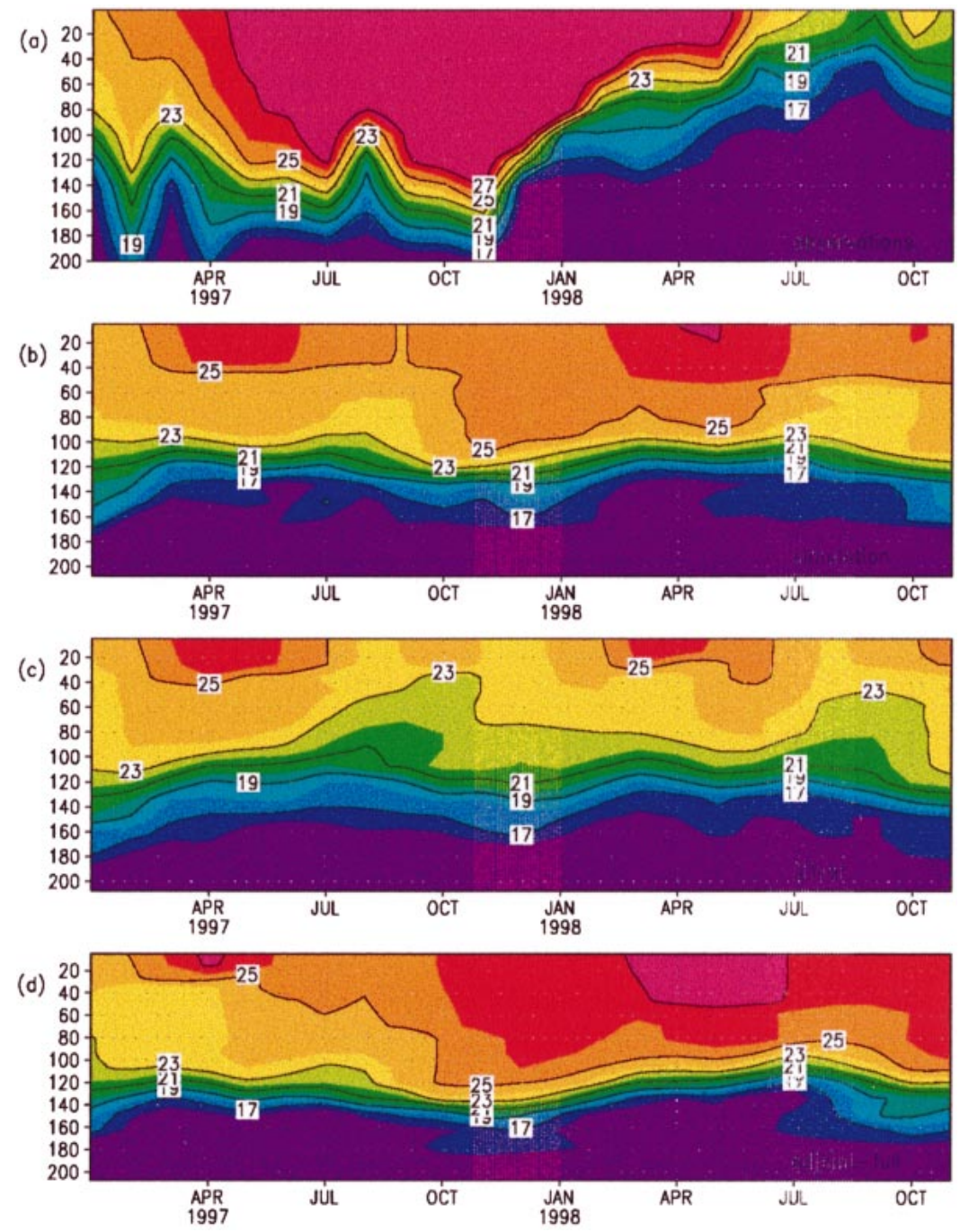

FIG. 7. The prediction of the $1997 / 98$ ENSO event (temperature) at $0^{\circ}, 140^{\circ} \mathrm{W}$ as a function of depth, using Jan initial conditions obtained from the (b) simulation run, (c) 3DVAR, and (d) adjoint assimilation. (a) The observed temperature.

3DVAR-based prediction has the coldest temperature, whereas the predicted temperature based on the adjoint initialization is the warmest (and thus closest to the observations).

We now turn to look at the predicted temperature anomalies of the 1997/98 event at the same location (Fig. 8), where the anomalies were calculated with respect to the seasonal prediction climatology of each prediction experiment. It can be seen that the 3DVARbased prediction hardly predicts an event, while the simulation-based prediction predicts a weak event, and the adjoint predicts a somewhat stronger event (although still much too weak, and the model also fails to switch from the El Niño event to the following La Niña).

These results demonstrate the importance of initializing the coupled model in a way that is consistent with the coupled model dynamics. The 3DVAR initialization results in model temperature initial conditions that are the closest to the observations of all three assimilation schemes used here. However, the model climatology has a bias, so its climatology is different from the observed one. As a result, initial conditions that correspond, for example, to the observed climatology with no anomaly, correspond in the model to the model climatology plus a strong artificial anomaly that is equal to the model bias. Now, the coupling to the atmosphere in the model is done via the SST anomalies, therefore the artificially strong model anomaly is amplified by the ocean-atmosphere coupling, and the resulting prediction skill is deteriorated. This error growth mechanism, a combination of the model climatological bias and instability dynamics, may be the reason for the differences between the 3DVAR-based prediction skill obtained with our model and the prediction skill obtained by Rosati et al. (1997), who used a higher-resolution model with a smaller climatological bias. The adjoint assimilation, on 

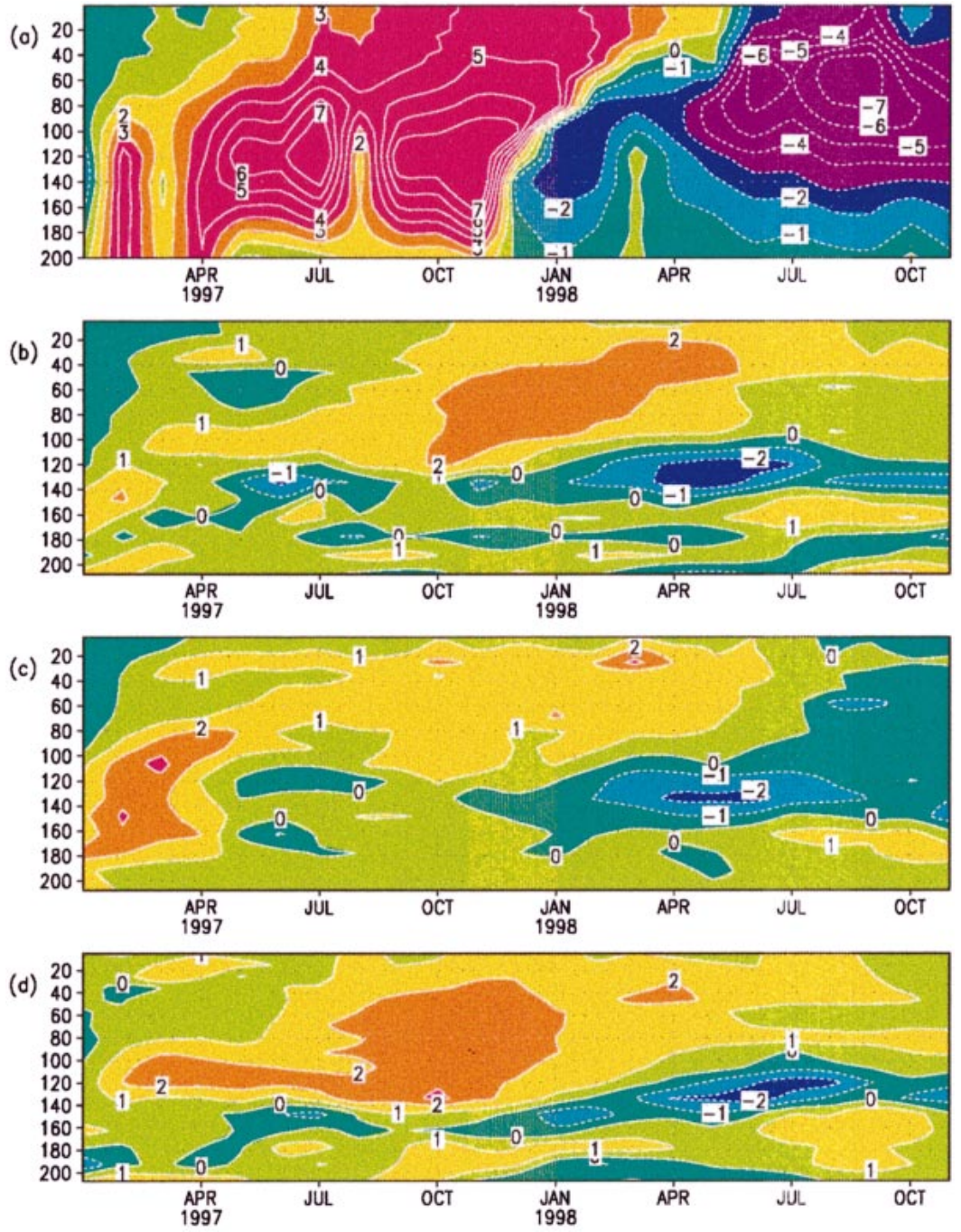

FIG. 8. Same as Fig. 7, but showing the temperature anomalies. The anomalies were calculated with respect to each type of prediction separately.

the other hand, takes into account the errors in the model dynamics and therefore allows only for the part of the observed temperature that is consistent with the model dynamics to influence the model trajectory. The initial conditions are therefore not as close to the observations, but the resulting predictability is somewhat better.

\section{c. Assimilation using the anomalous observed fields}

In both this subsection and the next one, we study various versions of the adjoint assimilation scheme. The prediction skill resulting from the different adjoint assimilation variants is shown in Figs. 9 and 10, and their predicted time series are shown in Figs. 2d-f and 4df. In the first variant to be considered here, we modify the cost function to be composed of the difference between the model anomalous fields (calculated with respect to the coupled model monthly climatology) and the observed anomalous fields (calculated with respect to their observed monthly climatology). Comparing the January prediction skill to that of the full-field assimilation (Fig. 9), we see that the initialization using the anomalous assimilation improved the prediction skill at time intervals of 3-6 months and is similar to the prediction skill based on the initialization using the fullfields assimilation during other prediction months. The above differences are clearly small and are even less clear in the July experiments (Fig. 10).

The motivation for the assimilation of the anomalous fields comes from the fact that the model climatology is somewhat different from that of the observed data. As explained at the end of section 5, this results in a deteriorated predictability. The adjoint method assimilation corrects this to some degree by not getting too close to the observed initial conditions, as explained above. However, this can be further corrected by assim- 


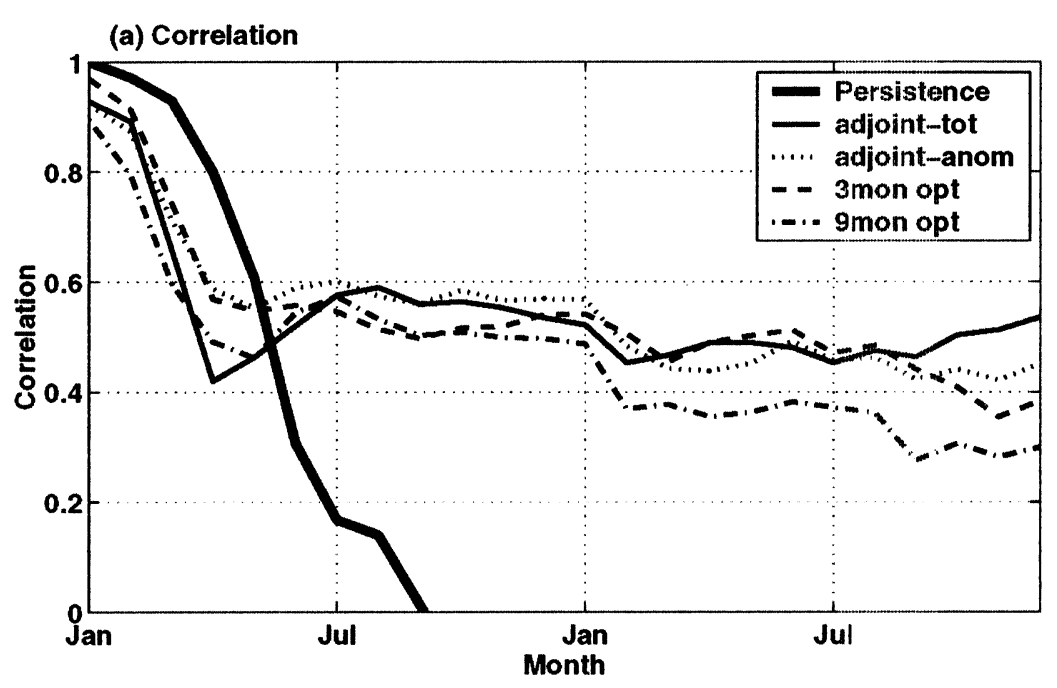

(b) RMS error

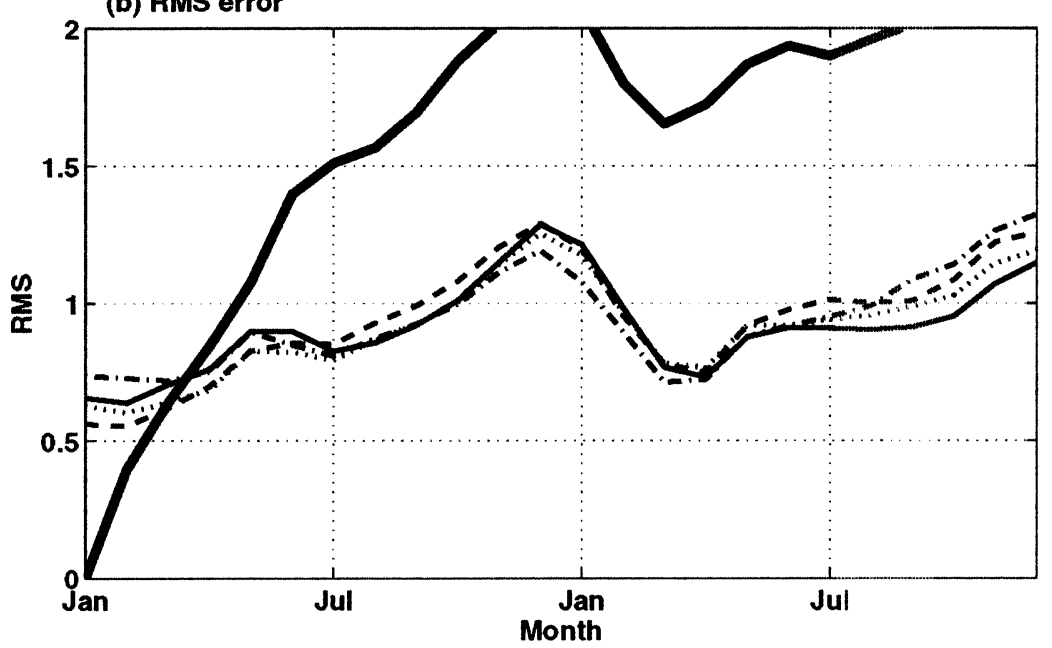

FIG. 9. Skill of the coupled model for the different adjoint initialization schemes. (a) Correlation between the predicted and the observed Niño-3 as function of the month of prediction. (b) The rms error of the predicted Niño-3.

ilating only the anomalies with respect to the corresponding climatologies of the model and the data. In this case, the model bias does not enter and does not cause the error growth described above. Assimilation of anomaly fields is routinely done with anomaly-based models (e.g., Chen et al. 1995), for which the assimilation of anomalies is required. But in hybrid coupled models and fully coupled models, only the full fields are normally assimilated. One may expect the assimilation of anomalies to improve prediction, in cases where climatological model bias causes it to deteriorate. Anomaly assimilation is not expected to correct predictability errors due to model bias in the variability rather than in the model climatology. That is, if the model ENSO events are too weak or occur in the wrong place, assimilating the anomalies will not improve the prediction skill.
The results of the anomaly assimilation are clearly not impressive here. It seems that in our model the bias due to the model climatology, which is corrected by the anomaly assimilation, is less significant than the bias in the model variability, which is not affected by the anomaly assimilation. We therefore expect that the anomaly assimilation using the adjoint method may actually have the potential to more significantly improve the prediction skill, in situations where the model variability bias is less than in our coarse model. A more thorough examination of the subject using higher-resolution models is needed.

\section{d. Optimization period}

It is not obvious how one should determine the time interval over which the assimilation should be per- 


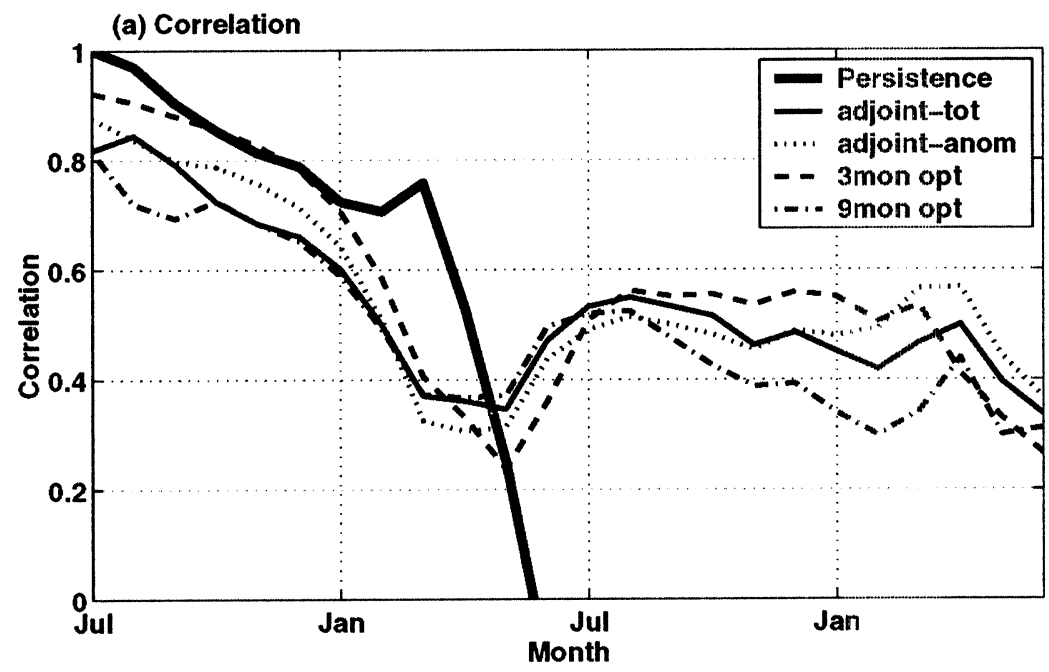

(b) RMS error

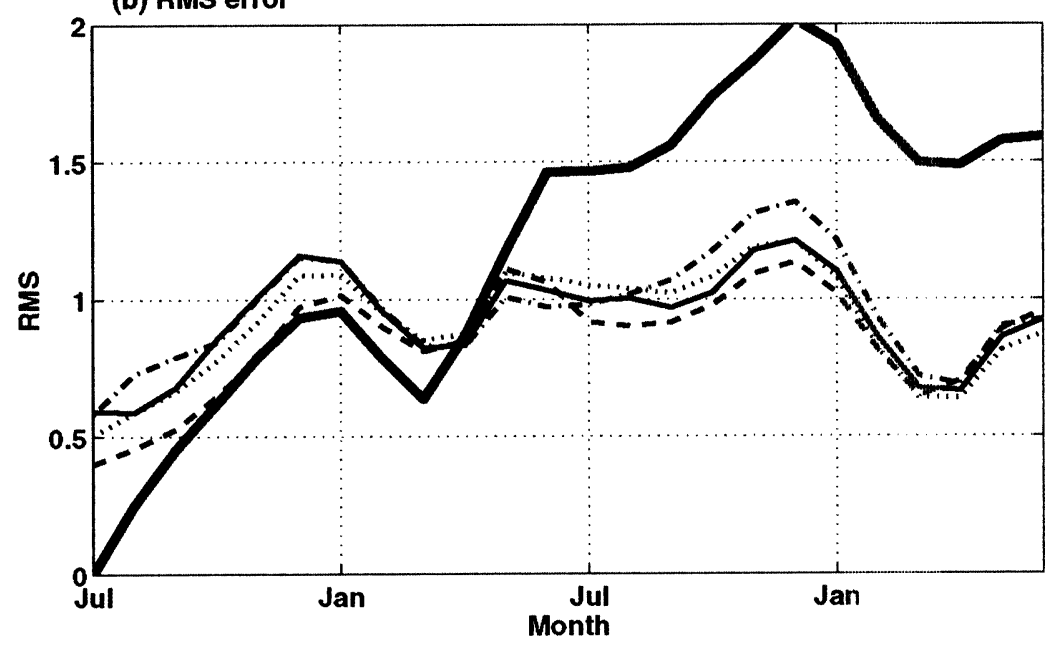

FIG. 10. Same as Fig. 9, but for prediction from Jul initial conditions.

formed; in general, a too short assimilation period would effectively turn the 4D assimilation into a 3D scheme, thus not taking advantage of the known model dynamics. Using a too long assimilation period, on the other hand, runs into the inherent predictability limits of the system. That is, whether ENSO is chaotic (Jin et al. 1994; Tziperman et al. 1994) or noise driven (Burgers 1999; Moore and Kleeman 1996; Penland and Sardeshmukh 1995), it has some inherent predictability timescale. If the assimilation period is longer than this timescale, then the initial conditions calculated at the beginning of the assimilation period cannot determine the state of the model at the end of the assimilation period. The linearization that underlies the adjoint formulation may also break down for such long assimilation periods. In this case, the initialization is clearly also meaningless for the prediction period. The standard time interval used in this study is 6 months. We now repeat the anom- aly adjoint assimilation of section $6 \mathrm{c}$, but with an assimilation period of 3 months (Figs. 2e and 4e). The prediction skill of prediction from January and July initializations are shown in Figs. 9 and 10 (dashed lines), respectively. In both seasons the initialization with 3month assimilation period is doing better during the first 6 months of the prediction, but at longer times the 6month assimilation has better skill. A 3-month assimilation period is not enough for the model dynamics to play a significant role, and the initial conditions obtained from the 3-month assimilation are therefore more affected by-and are closer to-the observations. This results in a better skill during the first few months of prediction since the model dynamics are less important then. At longer times (more than 6 months) the model dynamics come into play, and the 6-month initialization results in a better skill.

Next, we increase the optimization period to 9 months 
and initialize the coupled model with both January and July initial conditions (Figs. 9 and 10, dash-dotted lines). The prediction skill obtained with the 9-month optimization is similar to that of the 6-month optimization, especially at timescales longer than half a year. It seems, therefore, that an assimilation period of 6 months may be near optimal for our model. Lacking, however, a good theoretical understanding of what sets ENSO's predictability time, and therefore what should be the optimal assimilation period, we do not know how robust this result would be to model details such as resolution, etc.

\section{Conclusions}

In this study we investigated an experimental ENSO prediction system based on an ocean GCM coupled to a statistical atmosphere and the adjoint method of 4D variational data assimilation. The coupled model is initialized using the adjoint method in order to perform predictions from 1980 to 1999 . In order to compare the prediction skill of the system, two simpler assimilation techniques were also used to initialize the coupled model: forcing the ocean model with observed SST, wind stress, and surface fluxes, and the 3D variational assimilation method of Rosati et al. (1997). In addition, we examined the issue of the period over which the adjoint assimilation is to be conducted by initializing the model with assimilation periods of 3, 6, and 9 months and comparing the resulting prediction skill. Another issue examined here is the assimilation of the full observed fields versus the assimilation of the anomalous (with respect to model and observed monthly climatologies) fields.

A comparison between the skill of the coupled model when initialized with the adjoint method and the skill of the coupled model initialized using a forced ocean model and the 3DVAR method shows that the adjoint method does seem to have the potential of improving simpler assimilation schemes. The improved skill is mainly at prediction intervals of more than 6 months, when the coupled model dynamics start to influence the model solution. At shorter prediction time intervals, the initialization using the forced ocean model or the 3DVAR may result in a better prediction skill. This occurs because both simpler assimilation methods calculate initial conditions that are closer to the observed temperature at the beginning of the prediction period, while the adjoint method allows the initial conditions to deviate farther away from the observations, based on the model dynamics. In the first few months of the prediction, the initial conditions dominate the prediction skill, and therefore the simpler methods do better. It is also important to note that substantial discrepancies exist between our results for the 3DVAR initialization and those of Rosati et al. (1997), who used a higher-resolution ocean model and an atmospheric GCM. This raises important questions regarding the effects of the model resolution and of the atmospheric model used on the performance of the different assimilation schemes considered here, which will need to be addressed in future studies.

A detailed analysis of ENSO prediction experiments for the 1997/98 event, for which the adjoint method again performed somewhat better than the other two schemes considered here (although still not satisfactorily), strengthens the above conclusion regarding the potential of the adjoint method.

We explained that defining the cost function to be the difference between the observed and model anomalous fields (with respect to the model and observed climatologies, and instead of using the full fields) may eliminate the effects of systematic model bias and therefore improve predictive skill. In our experiments here, however, the assimilation of anomalies did not have a substantial effect on the prediction skill of the coupled model. This seems to indicate that in our model the bias due to the model climatology, which is corrected by the anomaly assimilation, is less significant than the bias in the model variability, which cannot be eliminated using the anomaly assimilation.

Changing the optimization period from 6 to 3 to 9 months showed that the period of 6 months seems to be a near optimal choice for our model. Shortening the optimization period to 3 months resulted in a better skill at short prediction times (up to 6 months) but reduced the skill at longer prediction times. Increasing the optimization interval to 9 months resulted in a reduced skill in all time intervals. Lacking a sufficient theoretical understanding of what sets ENSO's predictability limit, and therefore of the issue of optimal assimilation time, we cannot judge how robust these results are to model details and resolution.

All aspects of initialization and prediction studied here clearly need to be further investigated using higherresolution models in order to optimize the use of the adjoint method for ENSO prediction. This is especially relevant in view of the discrepancies between our results for the 3DVAR initialization and those of Rosati et al. (1997), who used a higher-resolution model and a 3DVAR. Nevertheless, we feel that it is reasonable to hope that the adjoint method may out perform other assimilation schemes at higher ocean resolutions, as it did at the coarse resolution used here. The results here therefore seem sufficiently encouraging to justify a further study.

Acknowledgments. Dedicated to the memory of Ziv Sirkes. We thank Geert Jan van Oldenborgh, Andrew Moore, and two anonymous reviewers for their most useful and constructive comments. This study was partially funded by the NOAA Office of Global Programs and by the Israeli Science Foundation. ZS was partially supported by the NOAA CLIVAR Atlantic program. 


\section{REFERENCES}

Ballabrera-poj, J., A. J. Busalacchi, and R. Murtugudde, 2001: Application of a reduced-order Kalman filter to initialize a coupled atmosphere-ocean model: Impact on the prediction of El Niño. J. Climate, 14, 1720-1737.

Behringer, D. W., M. Ji, and A. Leetmaa, 1998: An improved coupled model for ENSO prediction and implications for ocean initialization. Part I: The ocean data assimilation system. Mon. Wea. Rev., 126, 1013-1021.

Bennett, A. F., 1992: Inverse Methods in Physical Oceanography. Cambridge Monogr. on Mechanics and Mathematics. Cambridge University Press, 346 pp.

- , and P. C. McIntosh, 1982: Open ocean modeling as an inverse problem: Tidal theory. J. Phys. Oceanogr., 12, 1004-1018.

- B. S. Chua, D. E. Harrison, and M. J. McPhaden, 1998: Generalized inversion of tropical atmosphere-ocean data and a coupled model of the tropical Pacific. J. Climate, 11, 1768-1792.

Bonekamp, H., G. J. van Oldenborgh, and G. Burgers, 2001: Variational assimilation of tropical atmosphere-ocean and expendable bathythermograph data in the Hamburg ocean primitive equation ocean general circulation model, adjusting the surface fluxes in the tropical ocean. J. Geophys. Res., 106 (C8), 16693 16709 .

Burgers, G., 1999: The El Niño stochastic oscillator. Climate Dyn., 15, 521-531.

- M. A. Balmaseda, F. C. Vossepoel, G. J. van Oldenborgh, and P. J. van Leeuwen, 2002: Balanced ocean-data assimilation near the equator. J. Phys. Oceanogr., 32, 2509-2519.

Cane, M. A., S. E. Zebiak, and S. C. Dolan, 1986: Experimental forecasts of El Niño. Nature, 321, 827-832.

—, A. Kaplan, R. N. Miller, B. Tang, E. Hackert, and A. J. Busalacchi, 1996: Mapping tropical Pacific sea level: Data assimilation via a reduced state space Kalman filter. J. Geophys. Res., 101, 22 599-22 617

Chen, D., S. E. Zebiak, A. J. Busalacchi, and M. A. Cane, 1995: An improved procedure for El Niño forecasting: Implications for predictability. Science, 269, 1699-1702.

Derber, J., and A. Rosati, 1989: A global oceanic data assimilation system. J. Phys. Oceanogr., 19, 1333-1347.

Galanti, E., and E. Tziperman, 2003: A midlatitude-ENSO teleconnection mechanism via baroclinically unstable long Rossby waves. J. Phys. Oceanogr., 33, 1877-1888.

,-- M. Harrison, A. Rosati, R. Giering, and Z. Sirkes, 2002: The equatorial thermocline outcropping-A seasonal control on the tropical Pacific ocean-atmosphere instability strength. J. Climate, 15, 2721-2739.

Ghil, M., and P. Malanotte-Rizzoli, 1991: Data assimilation in meteorology and oceanography. Advances in Geophysics, Vol. 33 Academic Press, 141-266.

Gibson, J., S. Kallberg, S. Uppala, A. Nomura, A. Hernandez, and E. Serrano, 1997: ERA description. ECMWF Reanalysis Project Report Series 1, Tech. Rep. 1, ECMWF, Shinfield Park, Reading, United Kingdom, 72 pp.

Gill, P. E., W. Murray, and M. H. Wright, 1981: Practical Optimization. Academic Press, $401 \mathrm{pp}$.

Harrison, M. J., A. Rosati, B. J. Soden, E. Galanti, and E. Tziperman, 2002: An evaluation of air-sea flux products for ENSO simulation and prediction. Mon. Wea. Rev., 130, 723-732.

Jin, F.-F., D. Neelin, and M. Ghil, 1994: ENSO on the devil's staircase. Science, 264, 70-72.

Kalnay, E., and Coauthors, 1996: The NCEP/NCAR 40-Year Reanalysis Project. Bull. Amer. Meteor. Soc., 77, 437-471.

Kirtman, B. P., and S. E. Zebiak, 1997: ENSO simulation and prediction with a hybrid coupled model. Mon. Wea. Rev., 125, 2620-2641.

Kleeman, R., A. M. Moore, and N. R. Smith, 1995: Assimilation of subsurface thermal data into an intermediate tropical coupled ocean-atmosphere model. Mon. Wea. Rev., 123, 3103-3113.

Klinker, E., F. Rabier, G. Kelly, and J. F. Mahfouf, 2000: The ECMWF operational implementation of four-dimensional variational assimilation. III: Experimental results and diagnostics with operational configuration. Quart. J. Roy. Meteor. Soc., 126, 11911215

Latif, M., and N. E. Graham, 1992: How much predictive skill is obtained in the thermal structure of an OGCM? J. Phys. Oceanogr., 22, 951-962.

- and Coauthors, 1998: A review of the predictability and prediction of ENSO. J. Geophys. Res., 103 (C7), 14 375-14 393.

Lee, T., J. P. Boulanger, A. Foo, L. L. Fu, and R. Giering, 2000: Data assimilation by an intermediate coupled ocean-atmosphere model: Application to the 1997-1998 El Niño. J. Geophys. Res., 105 (C11), 26 063-26087.

Marotzke, J., 1992: The role of integration time in determining a steady state through data assimilation. J. Phys. Oceanogr., 22, 1556-1567.

McPhaden, M. J., and Coauthors, 1998: The Tropical Ocean-Global Atmosphere observing system: A decade of progress. J. Geophys. Res., 103 (C7), 14 169-14 240.

Miller, R. N., and M. A. Cane, 1989: A Kalman filter analysis of sea level heights in the tropical Pacific. J. Phys. Oceanogr., 19, 773790

Moore, A. M., and R. Kleeman, 1996: The dynamics of error growth and predictability in a coupled model of ENSO. Quart. J. Roy. Meteor. Soc., 122, 1405-1446.

Neelin, J. D., D. S. Battisti, A. C. Hirst, F.-F. Jin, Y. Wakata, T. Yamagata, and S. Zebiak, 1998: ENSO theory. J. Geophys. Res., 103 (C7), 14 261-14 290.

Pacanowski, R. C., and S. G. H. Philander, 1981: Parameterization of vertical mixing in numerical models of tropical oceans. $J$. Phys. Oceanogr., 11, 1443-1451.

—_, and S. M. Griffies, 1999: MOM 3.0 manual. NOAA/Geophysical Fluid Dynamics Laboratory, Princeton, NJ, 671 pp.

Penland, C., and P. D. Sardeshmukh, 1995: The optimal growth of tropical sea surface temperature anomalies. J. Climate, 8, 19992024.

Reynolds, R. W., and T. M. Smith, 1994: Improved global sea surface temperature analyses using optimum interpolation. J. Climate, 7, 929-948.

Rosati, A., K. Miyakoda, and R. Gudgel, 1997: The impact of ocean initial conditions on ENSO forecasting with a coupled model. Mon. Wea. Rev., 125, 752-772.

Sirkes, Z., E. Tziperman, and C. W. Thacker, 1996: Combining data and a global primitive equation ocean general circulation model using the adjoint method. Modern Approaches to Data Assimilation in Ocean Modeling, P. Malanotte-Rizzoli, Ed., Elsevier, 119-145.

Syu, H. H., and J. D. Neelin, 2000a: ENSO in a hybrid coupled model. Part I: Sensitivity to physical parameterizations. Climate Dyn., 16, 19-34.

— , and — 2000b: ENSO in a hybrid coupled model. Part II: Prediction with piggyback data assimilation. Climate Dyn., 16, 35-48.

Thacker, W. C., and R. B. Long, 1988: Fitting dynamics to data. J. Geophys. Res., 93, 1227-1240.

Tziperman, E., and W. C. Thacker, 1989: An optimal-control/adjointequations approach to studying the oceanic general circulation. J. Phys. Oceanogr., 19, 1471-1485.

,$- \ldots$, R. B. Long, and S. M. Hwang, 1992a: Oceanic data analysis using a general circulation model. Part I: Simulations. J. Phys. Oceanogr., 22, 1434-1457.

- $-\longrightarrow,-,-$, and S. R. Rintoul, 1992b: Oceanic data analysis using a general circulation model. Part II: A North Atlantic model. J. Phys. Oceanogr., 22, 1458-1485.

— - L. Stone, M. A. Cane, and H. Jarosh, 1994: El-Niño chaos: overlapping of resonances between the seasonal cycle and the Pacific ocean-atmosphere oscillator. Science, 264, 72-74.

van Oldenborgh, G., 2000: What caused the onset of the 1997-98 El Niño? Mon. Wea. Rev., 128, 2601-2607.

Weaver, A., J. Vialard, D. Anderson, and P. Delecluse, 2002: Three- 
and four-dimensional variational assimilation with a general circulation model of the tropical Pacific Ocean. ECMWF Tech. Rep. $365,74 \mathrm{pp}$.

Webster, P. J., 1995: The annual cycle and the predictability of the tropical coupled ocean-atmosphere system. Meteor. Atmos. Phys., 56, 33-35.

Zebiak, S. E., and M. A. Cane, 1987: A model El Niño-Southern Oscillation. Mon. Wea. Rev., 115, 2262-2278. 